\title{
RESPOSTAS ELETROCARDIOGRÁFICAS DE EQUINOS AO TREINAMENTO COM BASE NA CURVA VELOCIDADE-LACTATO DETERMINADA EM ESTEIRA ROLANTE
}

\author{
Raquel Mincarelli Albernaz, ${ }^{1}$ Deborah Penteado Martins Dias, ${ }^{1}$ Daniel Paulino Junior, ${ }^{1}$ \\ João Paulo da Exaultação Pascon, ${ }^{1}$ Antonio Queiroz-Neto ${ }^{2}$ e José Corrêa de Lacerda Neto ${ }^{1}$ \\ 1. Universidade Estadual Paulista, Faculdade de Ciências Agrárias e Veterinárias, Departamento de Clínica e Cirurgia Veterinária. \\ E-mail: raquel albernaz@hotmail.com \\ 2. Universidade Estadual Paulista, Faculdade de Ciências Agrárias e Veterinárias, Departamento de Morfologia e Fisiologia Animal.
}

\section{RESUMO}

Neste trabalho, avaliaram-se, mediante exame eletrocardiográfico, as adaptações cardiovasculares de equinos ao treinamento com base na curva velocidade-lactato. Para tanto, foram utilizados oito equinos da raça Puro Sangue Árabe previamente submetidos a um período de quatro meses de destreinamento. Os animais realizaram inicialmente um teste de esforço progressivo (ET), durante o qual colheram-se amostras de sangue para determinação do lactato. A velocidade na qual a concentração de lactato atingiu $4,0 \mathrm{mmol} / \mathrm{L}$ $\left(\mathrm{V}_{4}\right)$ foi calculada por meio de análise de regressão. Utilizaram-se $60 \%$ de $\mathrm{V}_{4}$ como velocidade de treinamento em esteira rolante, com duração de 45 minutos, três vezes por semana em dias alternados, durante oito semanas. Exames eletrocardiográficos (ECG) foram realizados antes e após o período de treinamento. $\mathrm{O}$ treinamento melhorou o condicionamento físico dos equinos, confirmado pelo aumento tanto de $\mathrm{V}_{2}$ como de $\mathrm{V}_{4}$ no segundo ET. Pelo exame ECG, observou-se aumento significativo na amplitude de onda $\mathrm{R}$ após o treinamento. Em que pese o treinamento ter melhorado a atividade metabólica, não foi suficiente para conferir modificações em parâmetros cardíacos, embora tenha promovido discretas mudanças no exame eletrocardiográfico.

PALAVRAS-CHAVES: Adaptações ao treinamento, cardiovascular, eletrocardiografia, esteira, equino.

\section{EQUINE ELECTROCARDIOGRAPHIC RESPONSES TO TRAINING BASED ON SPEED-LACTATE CURVE PERMORMED ON TREADMILL}

The aim of this study was to evaluate, by electrocardiographic analysis, equine cardiovascular adaptations to training based on speed-lactate curve. For this evaluation, eight Arabian horses previously submitted to four months of rest were used. The animals performed a graded exercise test (ET) and samples were collected for blood lactate measurement. The speed where the blood lactate concentration reached $4.0 \mathrm{mmol} / \mathrm{L}_{\left(\mathrm{V}_{4}\right) \text { was }}$ calculated by regression analysis. The estimated training speed was $60 \%$ of V4 performed on a treadmill for 45 min three times a week on alternate days for eight weeks. The electrocardiogram (ECG) analysis was made before and after the beginning of the training. The training improvement is confirmed by the increase in both $\mathrm{V}_{2}$ and $\mathrm{V}_{4}$ of the second ET. After the second ECG analysis, a significant increase in the $\mathrm{R}$ wave amplitude was observed. Training improved the metabolic activity, promoted discrete changes in electrocardiogram; however, it was not adequate to result changes in cardiac parameters.

KEYWORDS: Training adaptation, cardiovascular, electrocardiogram, treadmill, equine. 


\section{INTRODUÇÃO}

O coração é um órgão localizado na cavidade torácica, em sua posição anterior e ventral. Nos equinos, encontra-se em posicionamento característico, possui o ventrículo direito cranial à esquerda e está deslocado à esquerda da linha média torácica. Sua posição apical situa-se caudoventralmente no tórax, próxima ao osso esterno, à altura da porção dorsal do olécrano, ao passo que sua base está situada mais craniodorsalmente. Radiograficamente, o coração ocupa a extensão de cinco costelas e quatro espaços intercostais, da segunda à sétima costela (MENDES, 2004; REEF, 1998).

A principal função cardíaca é manter uma boa circulação sanguínea, a qual deve ser adequada para o bom funcionamento de todos os órgãos e tecidos do organismo. Se a demanda circulatória for aumentada, o coração poderá compensar mediante dois modos básicos possíveis pelos quais se proporciona maior volume circulante por minuto: aumento da frequência cardíaca e aumento da força de contração (MENDES, 2004).

O coração é um músculo e, como tal, responde e se adapta aos treinamentos na tentativa de manter a homeostase (MARLIN \& NANKERVIS, 2002; BUGAISKY et al., 1992). Qualquer mudança da demanda cardíaca, seja por atividade atlética ou alteração patológica, levará ao aumento ou à diminuição da massa cardíaca, denominados hipertrofia e atrofia cardíaca respectivamente (BUGAISKY et al., 1992).

Segundo GROSSMAN et al. (1975), existem dois tipos de adaptação em resposta ao treino de acordo com o tipo de miofibrila produzida. Pode-se ter um aumento das miofibrilas dispostas em série (hiperplasia), ampliando-se o diâmetro da câmara cardíaca, ou um aumento das miofibrilas dispostas em paralelo, expandindo-se a espessura do ventrículo esquerdo (hipertrofia). De acordo com os autores, existe a hipótese de que o aumento do diâmetro preceda o aumento da espessura cardíaca.

$\mathrm{O}$ aumento da espessura da parede favorece a força de contração, e o aumento da câmara cardíaca proporciona crescimento no volume de ejeção sanguínea (porcentagem de volume bombeado no final da sístole em cada batimento cardíaco). Isso contribui para um melhor desempenho do animal, pois mais sangue, e assim mais oxigênio, pode ser mandado para os órgãos e os tecidos (MARLIN \& NANKERVIS, 2002; POOLE \& ERICKSON, 2004).
Em equinos treinados, a massa cardíaca pode alcançar $1,1 \%$ do peso corporal e está relacionada com volume de ejeção, débito cardíaco e capacidade aeróbia. $\mathrm{O}$ débito cardíaco é definido como o volume de sangue bombeado dos ventrículos e pode ser mensurado em L/minutos Quando ocorre aumento do débito cardíaco, a demanda de oxigênio aos tecidos é favorecida, sendo que essa variável é a principal determinante da velocidade de distribuição de oxigênio máxima $\left(\mathrm{VO}_{2 \text { máx }}\right)$. Animais treinados podem obter valores de débito cardíaco superiores a $500 \mathrm{~L} / \mathrm{min}$ em virtude do aumento da massa cardíaca (POOLE \& ERICKSON, 2004).

A maior complicação relacionada a essa adaptação cardíaca, estudada in vivo, é o tamanho do coração comparado ao peso do animal. A hipertrofia cardíaca pode, em alguns casos, estar acompanhada de áreas necróticas, levando a desorganização miofibrilar, lesão mitocondrial e proliferação de tecido conectivo com acúmulo de colágeno (BUGAISKY et al., 1992).

A atividade elétrica do coração pode ser avaliada pelo exame eletrocardiográfico. Esse é um exame não invasivo, de baixo custo, de fácil realização e que pode fornecer informações sobre frequência e ritmo cardíaco, além de detectar anormalidades ou arritmias visíveis no traçado eletrocardiográfico (REEF, 1998). Em medicina esportiva equina utiliza-se o eletrocardiograma para a investigação de queda de desempenho atlético relacionada com fibrilação, bloqueio atrioventricular completo e contrações atrial e ventricular prematuras (EVANS, 1999).

Um dos fatores considerados importantes no formato dos diferentes tipos de ondas são as características para cada espécie. Em equinos, a onda P é geralmente bifásica ou polifásica e tem forma de "V", que não deve ser interpretada como anormalidade. A forma da onda $\mathrm{P}$ é, em parte, dependente da frequência cardíaca e, à medida que a frequência aumenta, o espaço entre os dois picos tende a ficar menor, ou mesmo desaparecer; a primeira parte da onda pode se perder à medida que a segunda seção se torna maior. Alterações na forma, independentemente da frequência, indicam que a origem da contração atrial está mudando (SPEIRS, 1999). Quando ocorrem alterações na ventilação ou perfusão, consideram-se os aumentos de ondas T, os quais devem ser diferenciados nos casos de hipercalemia. Variações em ondas T e no segmento ST podem ser interpretadas 
também como uma variação fisiológica relacionada ao treinamento (SPEIRS, 1999).

A onda $\mathrm{T}$ é o parâmetro mais variável de um eletrocardiograma em equinos. Quando os animais são submetidos a estresse ou exaustão física, é possível observar inversão de polaridade e aumento de amplitude que pode exceder o complexo QRS (STEEL et al., 1976; PICCIONE et al., 2003).

O estabelecimento de um programa de treinamento deve considerar a sobrecarga de esforço à qual serão submetidos os diferentes sistemas orgânicos (POWERS \& HOWLEY, 1997). As variáveis típicas que refletem a sobrecarga incluem a intensidade, a duração e a frequência do exercício (ISLER et al., 1982), além, obviamente, do desenvolvimento das habilidades próprias de cada modalidade.

Para orientar o estabelecimento desses princípios deve-se considerar a utilização de algumas variáveis úteis que avaliem o condicionamento e determinem a carga de trabalho dos animais. Dentre essas variáveis destaca-se o lactato, que tem sido o norteador de inúmeros programas de treinamento, sejam estes realizados em campo, pistas ou trilhas (WITTKE et al., 1994; COROUCÉ et al., 1997), ou em esteiras rolantes, sob condições controladas (WERKMANN et al., 1996; ETO et al., 2004). A curva estabelecida pelas concentrações sanguíneas de lactato determinadas em velocidades crescentes é denominada curva velocidade-lactato. A inclinação dessa curva reflete o padrão metabólico predominante em intervalos subsequentes de velocidades crescentes. Nas velocidades menores, há predomínio de metabolismo aeróbio e as concentrações de lactato se mantêm relativamente baixas. Entretanto, quando as exigências energéticas para atender velocidades crescentes alcançam valores elevados, o metabolismo predominante passa a ser o anaeróbio, havendo concomitantemente aumento marcante do lactato que se caracteriza por uma inflexão repentina da curva para cima. Esse ponto de inflexão é denominado limiar anaeróbio ou, o que parece ser a denominação mais adequada, início do acúmulo de lactato sanguíneo (BAYLY, 1986).

Dessa forma, objetivou-se com este estudo avaliar, por meio do eletrocardiograma, possíveis adaptações do sistema cardíaco de equinos ao treinamento físico realizado em esteira rolante com intensidade determinada pelos valores de lactato sanguíneo.

\section{MATERIAL E MÉTODOS}

Animais

Foram utilizados oito equinos adultos da raça Árabe, machos e fêmeas, com idades de cinco a seis anos, pesando entre 339 e $422 \mathrm{~kg}$, pertencentes ao rebanho experimental da Faculdade de Ciências Agrárias e Veterinárias da Unesp, câmpus de Jaboticabal. Os animais foram mantidos em piquetes de Tifton e receberam $40 \%$ da exigência energética para trabalho moderado (NRC, 1989) em concentrado comercial.

Antes do período experimental, submeteram-se os animais a exame físico para avaliação da higidez. Somente foram utilizados os equinos sadios, em boas condições nutricionais e que haviam passado por combate a endo e ectoparasitas e vacinação contra viroses respiratórias. Antes do início do trabalho, os animais permaneceram em repouso pelo período de pelo menos quatro meses. $\mathrm{O}$ casqueamento foi realizado previamente aos procedimentos experimentais e a cada cinco semanas, até o final do período experimental.

\section{Exame eletrocardiográfico}

O exame foi realizado com o animal em posição quadrupedal, sob superfície de borracha, contido em tronco tipo brete, localizado em ambiente calmo e silencioso.

Avaliou-se a atividade elétrica do coração mediante emprego do eletrocardiógrafo computadorizado de 12 canais $^{1}$ (modelo ECG - PC) na derivação base ápice com velocidade $25 \mathrm{~mm} / \mathrm{s}$ e sensibilidade $2 \mathrm{~N}$. Os eletrodos foram fixados à pele por meio de agulhas $21 \mathrm{G}$ de aço inoxidável, em região previamente umedecida com álcool. Obtiveram-se os traçados segundo a técnica descrita por FREGIN (1982).

Um trecho de aproximadamente dez minutos do traçado foi capturado e gravado em disco rígido de computador. Avaliaram-se frequência cardíaca; sequência de despolarização e repolarização miocárdica; morfologia; duração em segundos (seg.) da onda P; característica da polaridade da onda T; intervalos QT, PR e QRS; e amplitude em milivolts ( $\mathrm{mV}$ ) das ondas $\mathrm{R}, \mathrm{Q}$ e T.

1. Modelo de aquisição de ECG para computador (ECG - PC, Tecnologia Eletrônica Brasileira - TEB, Brasil). 
O mesmo procedimento foi realizado após oito semanas de treinamento sob as condições preestabelecidas.

Testes de esforço progressivo

Após três dias de adaptação a uma esteira rolante $^{2}$ de alto desempenho projetada para animais de grande porte, os cavalos foram considerados aptos à realização do teste de esforço progressivo, denominado exercício teste (ET). Antes do teste, os animais tiveram a veia jugular cateterizada utilizando o cateter ${ }^{3}$ tamanho $14 \mathrm{G}$, para permitir a colheita de sangue com o animal em movimento. Uma vez os equinos posicionados na esteira, esta foi acionada iniciando-se o ET. O teste consistiu em um aquecimento de cinco minutos a 1,7 $\mathrm{m} \cdot \mathrm{s}^{-1} \mathrm{e}$ cinco minutos a $3,5 \mathrm{~m} \cdot \mathrm{s}^{-1}$ sem inclinação. Após o período de aquecimento, a esteira foi elevada a $6 \%$ de inclinação, com velocidade inicial de $3,5 \mathrm{~m} \cdot \mathrm{s}^{-1} \mathrm{e}$, a cada três minutos, acrescentou-se $0,5 \mathrm{~m} \cdot \mathrm{s}^{-1}$ à velocidade anterior. $\mathrm{O}$ final do ET foi determinado pelo momento no qual cada animal demonstrou sinais de fadiga, como desconcentração, sudorese intensa, abaixamento da cabeça e cansaço, tendendo a não acompanhar a esteira rolante, deslocando-se para trás. Após esse período, o animal foi submetido a desaquecimento durante quinze minutos, a $1,7 \mathrm{~m} \cdot \mathrm{s}^{-1}$, objetivando sua recuperação.

As amostras de sangue para determinação do lactato sanguíneo foram depositadas em frascos ${ }^{4}$ contendo fluoreto de sódio $1 \%$ na proporção de $1: 2$, nos seguintes momentos: antes do teste (basal), ao final do aquecimento, trinta segundos antes do aumento de cada velocidade, trinta segundos antes e após o término do teste e do desaquecimento e sessenta minutos após o término do exercício. A determinação sanguínea de lactato foi realizada pelo método da lactato oxidase, com um analisador automático, ${ }^{5}$ e expressa em mmol/L.

Efetuaram-se os cálculos para determinação da velocidade na qual as concentrações de lactato correspondiam a 2,0 e 4,0 $\mathrm{mmol} / \mathrm{L}\left(\mathrm{V}_{4}\right)$ por meio de análise de regressão, a partir dos valores obtidos no ET (COUROUCÉ et al., 1997).

$\mathrm{O}$ cálculo de $\mathrm{V}_{4}$ foi utilizado no estabelecimento da carga de trabalho a ser realizada durante $o$

2 Esteira Galloper, Sahinco Ltda., Palmital, SP, BR.

3 Angiocatt, BD Ind. Cirúrgicas Ltda., Juiz de Fora, MG.

4 Vacuttainer, BD Ind. Ci úrgicas Ltda., Juiz de Fora, MG.

5 Lactímetro YSL 1500 Sport, Yellow Springs, Ohio, USA. treinamento, assim como na avaliação da eficiência do protocolo de treinamento. Os animais realizaram dois ET em esteira rolante: antes de iniciar o treinamento $\left(\mathrm{ET}_{1}\right)$ e após oito semanas de treinamento $\left(\mathrm{ET}_{2}\right)$.

\section{Treinamento}

Uma vez determinada a $\mathrm{V}_{4}$ nos exercícios testes, estabeleceu-se que a intensidade de trabalho a ser imposta aos animais corresponderia a $60 \%$ de $\mathrm{V}_{4}$, com duração de 45 minutos e frequência de três vezes por semana, em dias alternados. O programa de treinamento durou oito semanas, período em que os equinos realizaram um total de dezoito sessões de exercícios em esteira. Os animais foram submetidos a exames clínicos semanais para avaliação da integridade do sistema locomotor e higidez.

Análises estatísticas

Os diferentes parâmetros foram avaliados por meio de Análise de Variância (ANOVA). Com os valores médios pelo teste de comparação das médias (Teste t), com nível de significância igual a $\mathrm{P}<0,05$ (SAMPAIO, 2002), utilizando o programa SAS - Statistical Analysis Sistem (SAS 2002).

\section{RESULTADOS E DISCUSSÃO}

Os valores de $\mathrm{V}_{2}$ e $\mathrm{V}_{4}$ obtidos durante os testes de esforço progressivo realizados em esteira rolante, nas fases pré e pós-treinamento, estão relacionados na Figura 1. O treinamento melhorou o condicionamento físico dos equinos, o que foi confirmado pelos aumentos tanto de $\mathrm{V}_{2}$ como de $\mathrm{V}_{4}$ no segundo teste de esforço. O modelo de treinamento utilizado, com base nos valores de lactato obtidos no teste de esforço progressivo, também têm proporcionado aumento das variáveis estudadas em pesquisas de características semelhantes (GOMIDE et al., 2006).

$\mathrm{Na}$ Tabela 1 estão relacionadas as velocidades correspondentes a 2,0 $\left(\mathrm{V}_{2}\right)$ e $4,0 \mathrm{mmol} / \mathrm{L}$ de lactato sanguíneo $\left(\mathrm{V}_{4}\right)$, o valor para $60 \%$ de $\mathrm{V}_{4}$ e a velocidade necessária para o desenvolvimento de fadiga $\left(\mathrm{V}_{\text {máxima }}\right)$. $\mathrm{A} \mathrm{V}_{2}, \mathrm{a} \mathrm{V}_{4}$ e a velocidade de treinamento $\left(60 \%\right.$ de $\left.\mathrm{V}_{4}\right)$ apresentaram aumento significativo após o período de 
treinamento. Com relação à $\mathrm{V}_{\text {máxima }}$ não se observou diferença significativa no ET realizado após o treinamento.

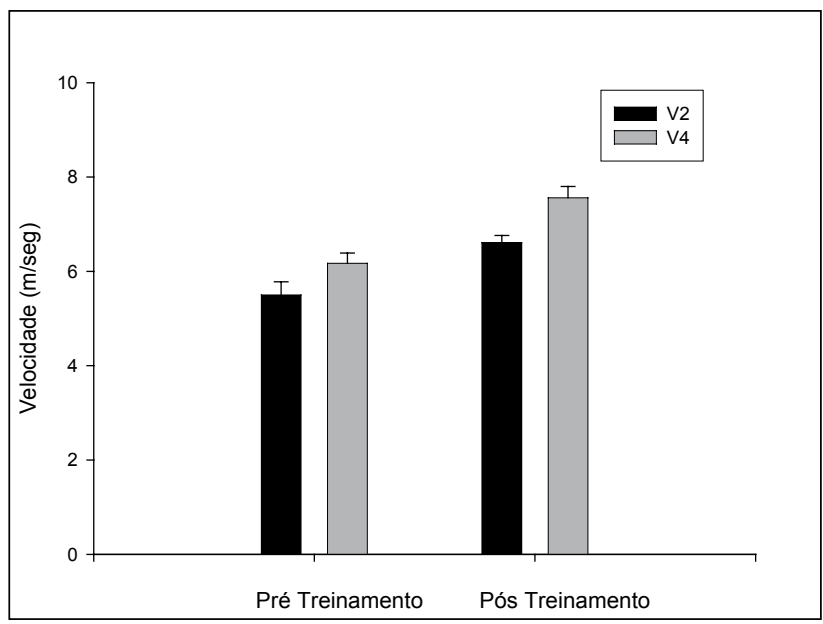

FIGURA 1. Médias \pm desvio padrão de velocidades correspondentes a $\mathrm{V}_{2}$ e $\mathrm{V}_{4}$, obtidas de equinos da raça Puro Sangue Árabe nas fases pré e pós-treinamento, durante teste de esforço progressivo realizado em esteira rolante $(* \mathrm{e} * *$ indicam valores maiores que os do pré-treinamento para mesma variável, $\mathrm{p} \leq 0,05$ ).

TABELA 1. Média \pm EPM dos valores determinados para a velocidade na qual a concentração de lactato atingiu $2,0 \mathrm{mmol} / \mathrm{L}$ $\left(\mathrm{V}_{2}\right), 4,0 \mathrm{mmol} / \mathrm{L}\left(\mathrm{V}_{4}\right), 60 \%$ do $\mathrm{V}_{4}$ e $\mathrm{V}_{\text {máxima }}$ durante exercício teste de equinos da raça Puro Sangue Árabe (PSA), antes e após o treinamento.

\begin{tabular}{lcc}
\hline Variável $(\mathrm{m} / \mathrm{seg})$ & Antes do treinamento & $\begin{array}{c}\text { Após o } \\
\text { treinamento }\end{array}$ \\
\hline $\mathrm{V}_{2}$ & $5,4 \pm 0,2$ & $6,4 \pm 0,3^{*}$ \\
$\mathrm{~V}_{4}$ & $6,3 \pm 0,3$ & $7,6 \pm 0,2^{*}$ \\
$60 \%$ de $\mathrm{V}_{4}$ & $3,8 \pm 0,2$ & $4,4 \pm 0,1^{*}$ \\
$\mathrm{~V}_{\text {máxima }}$ & $7,7 \pm 0,4$ & $8,2 \pm 0,3$ \\
\hline
\end{tabular}

*Valor maior que o obtido antes do treinamento pelo teste de Tukey $(\mathrm{p} \leq 0,05)$.

O programa de treinamento delineado para os eqüinos, neste trabalho com a carga de esforço correspondente a $60 \%$ de $\mathrm{V}_{4}$, produziu aumento significativo tanto da $\mathrm{V}_{2}$ como da $\mathrm{V}_{4}$. Outros trabalhos mostram a importância de se adotar a velocidade em que a concentração sanguínea de lactato atinge $4,0 \mathrm{mmol} / \mathrm{L}\left(\mathrm{V}_{4}\right)$ durante o teste de esforço como forma de avaliação do condicionamento de equinos (GERARD et al., 2002; ART \& LEKEUX, 1993). Neste experimento, houve aumento de $21,0 \%$ na $\mathrm{V}_{4}$ após o treinamento, superior ao relatado por TRILK et al. (2002), que observaram elevação de $17 \%$ nessa variável em treinamento também guiado pelos resultados da curva velocidade-lactato e desenvolvido em esteira rolante. Outro trabalho, utilizando a curva velocidade-lactato para treinar equinos em esteira, não logrou êxito ao procurar elevações na $\mathrm{V}_{4}$ após dois meses de treinamento, quando a duração das sessões de exercícios foi de apenas cinco ou quinze minutos (WERKMANN et al., 1996).

$\mathrm{O}$ aumento da $\mathrm{V}_{4}$ observado no decorrer do treinamento deve-se às adaptações metabólicas decorrentes do programa adotado. Segundo EATON et al. (1999), GERARD et al. (2002), HINCHCLIFF et al. (2002) e McGOWAN et al. (2002), o aumento da $\mathrm{V}_{4}$ e as menores concentrações de lactato sob a mesma intensidade de exercício no decorrer do treinamento indicam maior produção energética por via aeróbia. Esta decorre do melhor aproveitamento de lactato, segundo DONOVAN \& BROOKS (1983), e de gordura, segundo MUÑOZ et al. (2002), como fontes energéticas, assim como maior atividade das enzimas do metabolismo aeróbio e do sistema tampão das fibras musculares (McGOWAN et al., 2002). Aumento na atividade da citrato sintetase, a enzima envolvida na entrada de acetil-CoA no ciclo dos ácidos tricarboxílicos, foi verificado em equinos após o treinamento (HODGSON et al., 1985). Considerando que as vias enzimáticas de produção de energia presentes na fibra muscular estão estreitamente inter-relacionadas, o aumento na atividade da citrato sintetase implicaria em aumento de atividade de outras enzimas envolvidas no ciclo mencionado. Aparentemente, os progressivos aumentos na intensidade do treinamento constituem o estímulo necessário para induzir contínuo aumento de atividade enzimática nas fibras musculares. Esse aumento permitiria que uma maior proporção de energia se originasse dos mecanismos aeróbios, os quais são mais eficientes que as vias anaeróbias (EATON et al., 1999).

Treinamentos realizados em velocidades menores e com duração de 45 minutos provocam maiores alterações musculares (MARTINS et al., 2007) e, consequentemente, mais efeitos positivos na capacidade de resistência do que quando o treinamento tem 
maior intensidade e menor duração (GANSEN et al., 1999). Neste experimento, sessões submáximas com intensidades baseadas na $\mathrm{V}_{4}$ e com 45 minutos de duração provocaram estímulo suficiente para aumentar a capacidade de resistência dos equinos. Apesar de TRILK et al. (2002) indicarem períodos de duas semanas para o ajuste da intensidade do treinamento buscando aperfeiçoar o incremento do $\mathrm{V}_{4}$, neste caso, mesmo sem a realização de ajuste, o treinamento durante oito semanas foi suficiente para levar a um expressivo aumento nessa variável.

Ainda que McGOWAN et al. (2002) tenham encontrado concentrações de lactato maiores no plasma e menores nos músculos no momento da fadiga, após um período de treinamento, demonstrando que essa variável aumenta a capacidade de tamponamento e de remoção do lactato muscular, não se observaram, com o treinamento, alterações nas concentrações de lactato determinadas ao final dos testes progressivos e após o período de desaquecimento. HINCHCLIFF et al. (2002) também não encontraram, em dez semanas de treinamento, diferença significativa na concentração de lactato ao final do teste. Os dados obtidos se justificariam, portanto, por uma intensidade de exercício insuficiente para induzir alterações significativas (ART \& LEKEUX, 1993). Contudo, considerando que os valores de lactato obtidos durante os testes de esforço apresentam grande variabilidade individual (RONÉUS et al., 1994), aspecto detectado durante esta avaliação, poderia ser esse o fator que dificultou a obtenção de resultados significativos na análise estatística.

A velocidade necessária para a manifestação de fadiga $\left(\mathrm{V}_{\text {máxima }}\right)$ durante os testes em esteira aumentou ao final de noventa dias de treinamento. Esse dado mostra que o metabolismo dos animais sofreu adaptações que possibilitaram a manutenção do exercício por mais tempo, melhorando o desempenho (LINDNER, 1997). $\mathrm{O}$ aumento do tempo necessário para a manifestação de fadiga com o treinamento já foi relatado por outros autores (GERARD et al., 2002; McGOWAN et al., 2002) e pode ser um parâmetro para avaliar a melhoria no condicionamento físico (McGOWAN et al., 2002).

TRILK et al. (2002) relataram, na fase final de uma pesquisa realizada com equinos treinados em esteira, indícios de alterações psicológicas típicas de sobretreinamento e caracterizaram a falta de disposição e a diminuição na ingestão de alimentos como sendo os sinais de distúrbio. No entanto, os animais utilizados neste trabalho mostraram excelente disposição durante as sessões de exercícios e mantiveram o apetite. Atribui-se essa resposta ao fato de os equinos utilizados neste trabalho terem permanecido a campo, em grupo, com a oportunidade não apenas de manter o relacionamento social, tão importante nessa espécie, como também de diversificar a rotina do treinamento por meio de atividades de lazer, no piquete.

Os dados referentes ao exame eletrocardiográfico estão apresentados na Tabela 2.

TABELA 2. Média \pm desvio padrão de variáveis eletrocardiográficas determinadas em equinos da raça Puro Sangue Árabe em repouso antes e após oito semanas de treinamento em esteira rolante

\begin{tabular}{lcc}
\hline Variáveis & $\begin{array}{c}\text { Antes do } \\
\text { treinamento }\end{array}$ & $\begin{array}{c}\text { Após o } \\
\text { treinamento }\end{array}$ \\
\hline Frequência cardíaca (bpm) & $37,38 \pm 1,66$ & $35,13 \pm 1,92$ \\
Duração de P (seg) & $0,134 \pm 0,005$ & $0,137 \pm 0,013$ \\
Amplitude de P (mV) & $0,210 \pm 0,020$ & $0,310 \pm 0,040$ \\
Intervalo P-R (seg) & $0,339 \pm 0,011$ & $0,319 \pm 0,022$ \\
Duração QRS (seg) & $0,147 \pm 0,002$ & $0,146 \pm 0,008$ \\
Amplitude de R- (mV) & $1,510 \pm 0,100$ & $2,040 \pm 0,130^{*}$ \\
Intervalo Q-T (seg) & $0,531 \pm 0,009$ & $0,517 \pm 0,009$ \\
Amplitude de Q (mV) & $0,410 \pm 0,110$ & $0,310 \pm 0,070$ \\
\hline
\end{tabular}

*Valor maior que o obtido antes do treinamento pelo teste de Tukey $(\mathrm{p} \leq 0,05)$.

Os valores de frequência cardíaca observados se encontram dentro dos intervalos fisiológicos relatados para animais da espécie equina em inatividade (FERNANDES et al., 2004). Os valores obtidos após o treinamento não diferiram estatisticamente dos registrados antes do treinamento, porém, houve tendência à diminuição, o que corrobora a afirmação de que cavalos treinados - e consequentemente bem condicionados fisicamente - apresentam frequência cardíaca inferior à de equinos não condicionados (LITTLEJOHN, 1987).

O processo de despolarização no miocárdio ventricular pode ser afetado pelo treinamento intensivo e provavelmente por ação direta da duração da diástole e por fatores que interferem nssa duração, incluindo os efeitos autonômicos e possivelmente os efeitos metabólicos nas fibras miocárdicas influenciados pela 
circulação coronariana (HOLMES \& REZAKHANI, 1975). Neste estudo, houve diferença estatística na amplitude de onda $\mathrm{R}$, sugerindo aumento no tempo de despolarização dos ventrículos, o que pode estar relacionado à hipertrofia ou à hiperplasia da câmara cardíaca, em função do treinamento.

SKARDA et al. (1976) avaliaram a influência do treinamento sobre a frequência cardíaca, a duração dos intervalos PQ e QT e do complexo QRS em equinos da raça Puro Sangue Inglês. A análise foi realizada antes e após seis semanas de exercício submáximo em pista de areia. Neste estudo, não se observaram alterações significativas do traçado eletrocardiográfico que comprovassem uma melhora do estado atlético com o treinamento.

$\mathrm{O}$ efeito do treinamento sobre os parâmetros eletrocardiográficos foi avaliado por YONEZAWA et al. (2009) utilizando oito éguas da raça Puro Sangue Árabe, submetidas a treinamento de vinte dias em esteira de alta velocidade. Realizaram-se a análise e a interpretação dos traçados eletrocardiográficos quanto a ritmo, frequência cardíaca, duração e amplitude das ondas e intervalos. Neste ensaio, foi observada somente uma diminuição da frequência cardíaca de repouso após o treinamento, a qual foi atribuída ao manejo dos animais durante o período experimental.

A onda T, segundo SEVESTRE (1982), compreende um parâmetro variável; sua interpretação pode ser difícil, devido a variações de forma e amplitude. Neste estudo, quanto à característica de polaridade, a onda $\mathrm{T}$ permaneceu bifásica ao início do período experimental, assim como após oito semanas de treinamento, em todos os animais avaliados no exame proposto. Em estudo eletrocardiográfico de equinos da raça Mangalarga Marchador realizado por DINIZ et al. (2008), a onda $\mathrm{T}$ foi positiva em onze $(18,3 \%)$, negativa em doze (20\%) e bifásica em 37 (61,7\%) dos casos. Segundo os autores, tal distribuição para as ondas foi considerada compatível com o padrão descrito por PATTESON (1996) para essa derivação e, portanto, semelhante à de outras raças.

Tem sido postulado que alguns tipos de arritmias, como o bloqueio atrioventricular de segundo grau detectado em equinos em repouso, desaparecem durante o exercício (HOLMES \& ALPS, 1966). Neste trabalho, um dos cavalos estudados apresentou em repouso bloqueio atrioventricular de segundo grau no ECG realizado antes do treinamento; porém, essa arritmia desapareceu após o treinamento. A interrupção do batimento observado no bloqueio atrioventricular de segundo grau é uma resposta ao aumento gradual do pico de pressão aórtica (HOLMES, 1982). Considerando que essa é provavelmente uma resposta baropressórica, o esforço produzido durante o treinamento, associado a possíveis aumentos da pressão arterial durante exercícios de maior intensidade, como ocorreu neste trabalho, causaria ajuste na sensibilidade dos receptores de pressão e, consequentemente, desaparecimento do bloqueio mencionado.

\section{CONCLUSÃO}

Embora o treinamento em esteira rolante, nas condições do presente estudo, tenha melhorado as variáveis associadas à atividade metabólica dos equinos, não foi suficiente para causar modificações na maioria dos parâmetros eletrocardiográficos, com exceção da amplitude da onda $\mathrm{R}$.

\section{REFERÊNCIAS}

ART, T.; LEKEUX, P. Training-induced modifications in cardiorespiratory and ventilation measurements in Thoroughbred horses. Equine Veterinary Journal, v. 25, p. 532-536, 1993.

BAYLY, W. M. Blood lactate measurements. Anais International Conference for Equine Sports Medicine Veterinarians. San Diego, California. 1986. p. 54-55.

BUGAISKY, B. L.; GUPTA, M.; GUPTA, M. P.; ZAK, R. Cellular and molecular mechanisms of cardiac hypertrophy. In: FOZZARD, H. A.; HABER, A.; JENNINGS, R. B.; KATZ, A. M.; MORGAN, H. I. (Eds.). The heart and cardiovascular system. $2^{\text {nd }}$ ed. New York: Raven Press, 1992. p. 1.621-1.640.

DINIZ, M. P.; MUZZI, R. A. L.; MUZZI, L. A. L.; ALVES, G. E. S. Estudo eletrocardiográfico de equinos da raça Mangalarga Marchador. Arquivo Brasileiro de Medicina Veterinária e Zootecnia, v. 60, n. 3, p. 536-542, 2008.

DONOVAN, C. M.; BROOKS, G. A. Endurance training affects lactate clearance, not lactate production. American Journal of Physiology, v. 244, p. E83-E92, 1983.

EATON, M. D.; HODGSON, D. R.; EVANS, D. L.; ROSE, R. J. Effects of low- and moderate-intensity training on metabolic responses to exercise in Thoroughbreds. Equine Veterinary Journal, Suppl. Supl. 30, p. 521-527, 1999. 
ETO, D.; HADA, T.; KUSANO, K.; KAI, M.; KUSUNOSE, R. Effects of three kinds of severe repeated exercises on blood lactate concentrations in Thoroughbred horses in a treadmill. Journal of Equine Science, v. 15, n. 3, p. 61-65, 2004.

EVANS, D. L. Cardiac responses to exercise and training. In: MARR, C.M. Cardiology of the horse. Philadelphia: W. B. Saunders, 1999. p. 32-46.

FERNANDES, W. R.; LARSSON, M. H. M. A.; ALVES, A. L. G. et al. Características eletrocardiográficas em equinos clinicamente normais da raça Puro Sangue Inglês. Arquivo Brasileiro de Medicina Veterinária e Zootecnia, v. 56, p. 143-149, 2004.

FREGIN, G. F. The equine electrocardiogram with standardized body and limb positions. Cornell Veterinarian, v. 72, p. 304-324, 1982.

GANSEN, S.; LINDNER, A.; MARX, S.; MOSEN, H.; SALLMANN, H. P. Effects of conditioning horses with lactate-guided exercise on muscle glycogen content. Equine Veterinary Journal, v. 30, p. 329-331, 1999.

GERARD, M. P.; HODGSON, D. R.; LAMBERTH, R. R.; RAY, S. P.; ROSE, R. J. Effects of somatotropin and training on indices of exercise capacity in Standardbreds. Equine Veterinary Journal, v. 34, p. 496-501, 2002.

GOMIDE, L. M. W.; MARTINS, C. B.; OROZCO, C. A.; SAMPAIO, R. C. L.; BELLI, T.; BALDISSERA, V.; LACERDANETO, J. C. Concentrações sanguíneas de lactato em equinos durante a prova de fundo do concurso completo de equitação. Ciência Rural, v. 36, n. 2, p. 509-513, 2006.

GROSSMAN, W.; JONES, D.; McLAURIN, L. P. Wall stress and patterns of hypertrophy in the human left ventricle. Journal of Clinical Investigation, v. 56, p. 56-64, 1975.

HINCHCLIFF, K. W.; LAUDERDALE, M. A.; DUTSON, J.; GEOR, R. J.; LACOMBE, V. A.; TAYLOR, L. E. High intensity exercise conditioning increases accumulated oxygen deficit of horses. Equine Veterinary Journal, v. 34, p. 9-16, 2002.

HODGSON, D. R.; ROSE, R. J.; DIMAURO, J.; ALLEN, J. R. Effects of a submaximal treadmill training programme on histochemical properties, enzyme activities, and glycogen utilization of skeletal muscle in the horse. Equine Veterinary Journal, v. 17 , p. $300-305,1985$.

HOLMES, J. R. A superb transport system: the circulation. Equine Veterinary Journal, v. 14, p. 267-302, 1982.

HOLMES, J. R.; ALPS, B. J. The effect of exercise on rhythm irregularities in the horse. Veterinary Record, v. 78, p. 672-676, 1966.
HOLMES, J. R.; REZAKHANI, A. Observations on the T wave of the equine electrocardiogram. Equine Veterinary Journal, v.7, p.55-62, 1975.

ISLER, R.; STRAUB, R.; APPENZELLER, T.; GYSIN, J. Beurteilung der aktuellen Leistungsfähigkeit zur Fastlegung der optimalen Belastungsintensitat fur Intervalltraining bei Warmblutpferden. Schweizer Archiv fur Tierheilkunde, v. 123, p. 603-612, 1982.

LINDNER, A. Biochemical variables of energy metabolism in blood or plasma. In: Performance diagnosis of horses. Germany: Wageningen Pers, 1997. p. 8-28.

LITTLEJOHN, A. Exercise-related cardiovascular problems. In: ROBINSON, N. E. (Ed.). Current therapy in equine medicine 2. Philadelphia: W. B. Saunders, 1987. p. 176-180.

MARLIN, D.; NANKERVIS, K. Exercise and training responses. In: 2002. p. 113-126. Equine exercise physiology. Oxford: Blackwell,

MARTINS, C. B.; OROZCO, C. A. G.; GOMIDE, L. M. W.; SILVA, M. A. G.; CHRISTOVÃO, F. G.; QUEIROZ NETO, A.; LACERDA NETO, J. C. Efeito do condicionamento atlético sobre o músculo glúteo médio de equinos da raça puro sangue árabe. ARS Veterinária, v. 23, p. 100-107, 2007.

McGOWAN, C. M.; GOLLAND, L. C.; EVANS, D. L.; HODGSON, D. R.; ROSE, R. J. Effects of proloned training, overtraining and detraining on skeletal muscle metabolites and enzymes. Equine Veterinary Journal, v. 34, Supl.??? p. 257-263, 2002.

MENDES, D. N. Semiologia do sistema circulatório de equinos e ruminantes. In: FEITOSA, F. L. F. Semiologia veterinária: a arte do diagnóstico: cães, gatos, equinos, ruminantes e silvestres. São Paulo: Rocca, 2004. p. 234- 277.

PATTESON, M. W. Equine cardiology. Oxford: Blackwell Science, 1996. 254 p.

PICCIONE, G.; ASSENZA, A.; FAZIO, F.; GIUDICE, E.; CAOLA, G. Electrocardiographic changes induced by physical exercise in the jumper horse. Arquivo Brasileiro de Medicina Veterinária e Zootecnia, v. 55, n. 4, p. 397-404, 2003.

POOLE, D. C.; ERICKSON, H. Heart and vessels: function during exercise and response to training. In: HINCHCLIFF, K. W.; KANEPS, A. J.; GEOR, R. J. Equine sports medicine and surgery. Ohio: Saunders, 2004. p. 699-728.

POWERS, S. R.; HOWLEY, E. T. Fisiologia do exercício: teoria e aplicação ao condicionamento e ao desempenho. 3. ed. São Paulo: Manole, 1997. 527 p. 
REEF, V. B. Cardiovascular ultrasonography. In: Equine veterinary ultrasound. Philadelphia: W. B. Saunders Company, 1998.

RONÉUS, N.; ESSEN-GUSTAVSSON, B.; LINDHOLM, A.; ERIKSSON, Y. Plasma lactate response to submaximal and maximal exercise tests with training, and its relationship to performance and muscle characteristics in Standardbred trotters. Equine Veterinary Journal, v. 26, p. 117-121, 1994.

SAMPAIO, I. B. M. Estatística aplicada à experimentação animal. 2. ed. Belo Horizonte: Fundação de Ensino e Pesquisa em Medicina Veterinária e Zootecnia, 2002.

SAS. Statistical Analysis System Institute. SAS User's guide: Statistics. Cary: SAS, 2002.

SEVESTRE, J. A eletrocardiografia no cavalo. A Hora Veterinária, v. 2, p. 28-36, 1982.

SKARDA, R. T.; MUIR, W. W.; MILNE, D. W. et al. Effects of training on resting and postexercise ECG in Standardbred horses, using a standardized exercise test American Journal of Veterinary Research, v. 37, p. 1.485-1.488, 1976.

SPEIRS, V. C. O sistema cardiovascular. In: Exame clínico
STEEL, J. D.; HALL, M. C.; STEWART, G. A. Cardiac monitoring during exercise tests in the horse. Australian Veterinary Journal, v. 52, p. 6-10, 1976.

TRILK, J. L.; LINDNER, A. J.; GREENE, H. M.; ALBERGHINA, D.; WICKLER, S. J. A lactate-guided conditioning programme to improve endurance performance. Equine Veterinary Journal, v. 34, p. 122-125, 2002.

WITTKE, P.; LINDNER von, A.; DEEGEN, E.; SOMMER, H. Effects of training on blood-lactate running speed relationship in Thoroughbred racehorses. Journal of Applied Physiology, v. 77, p. 298-302, 1994.

WERKMANN, J.; LINDNER, A.; SASSE, H. H. L. Conditioning effects in horses of exercise of 5, 15 or 25 minutes duration at two blood lactate concentrations. Pferdeheilkunde, v. 12, p. 474-479, 1996.

YONEZAWA, L. A.; MACHADO, L. P.; SILVEIRA, V. F.; WATANABE, M. J.; SAITO, M. E.; KITAMURA, S. S.; KOHAYAGAWA, A. Exame eletrocardiográfico em equinos da raça puro sangue árabe submetidos ao exercício em esteira de alta velocidade e à suplementação com vitamina E. Archives of Veterinary Sciences, v. 14, n. 3, p. 134-142, 2009. dos equinos. Porto Alegre: Artmed, 1999. p. 183-206. 